

\section{DISCLAIMER}

This report was prepared as an account of work sponsored by an agency of the United States Government. Neither the United States Government nor any agency thereof, nor any of their employees, make any warranty, express or implied, or assumes any legal liability or responsibility for the accuracy, completeness, or usefulness of any information, apparatus, product, or process disclosed, or represents that its use would not infringe privately owned rights. Reference herein to any specific commercial product, process, or service by trade name, trademark, manufacturer, or otherwise does not necessarily constitute or imply its

endorsement, recommendation, or favoring by the United States Government or any agency thereof. The views and opinions of authors expressed herein do not necessarily state or reflect those of the United States Government or any agency thereof. 


\section{DISCLAIMER}

Portions of this document may be illegible in electronic image products. Images are produced from the best available original document. 


\title{
SPIN-FLOP TENDENCIES IN EXCHANGE-BIASED Co/CoO THIN FILMS
}

\author{
J.A. Borchers, Y. Ijiri, S.-H. Lee, and C.F. Majkrzak \\ National Institute of Standards and Technology \\ Gaithersburg, Maryland 20899 \\ G.P. Felcher \\ Argonne National Laboratory \\ Argonne, Illinois, 60439 \\ Fentaro Takano, R.H. Kodama, and A.E. Berkowitz \\ Center for Magnetic Recording Research, University of California at San Diego \\ La Jolla, California, 94720
}

\begin{abstract}
In order to study the antiferromagnetic (AFM) spin structure near the interface of exchange-biased bilayers, polarized neutron diffraction measurements were performed on a series of $(111) \mathrm{Co}(7.5 \mathrm{~nm}) / \mathrm{CoO}(\mathrm{X} \mathrm{nm})$ and $\mathrm{CoO}(\mathrm{X}$ $\mathrm{nm}$ ) thin films where $X=20,40$ and $100 \mathrm{~nm}$. In these samples, field cooling through the Néel temperature of the AFM increases the component of the $\mathrm{CoO}$ moment " perpendicular to the applied field, relative to the parallel component. The subsequent application of a 500 Oe field perpendicular to the cooling direction rotates both the $\mathrm{Co}$ and $\mathrm{CoO}$ moments. Experiments on $\mathrm{CoO}$ films without Co showed a smaller difference between the parallel and perpendicular $\mathrm{CoO}$ moments in response to cooling and applied fields. Ex-
\end{abstract}


change coupling between the $\mathrm{Co}$ and $\mathrm{CoO}$ layers is apparently responsible for the increased projection of the AFM moments perpendicular to the cooling field. 
The term "exchange-biasing" describes a phenomenon associated with interfacial coupling between ferromagnetic (FM) and antiferromagnetic (AFM) materials. In particular, a shift of the magnetic hysteresis loop along the field axis is observed after field cooling these composites through the Neel temperature of the AFM. Since its discovery over 40 years ago in oxidized Co particles., a number of experimental studies ${ }^{2-6}$ have focused on layered systems because of their potential applications in magnetic sensors. ${ }^{\top}$ Still unresolved, however, is the link between exchange biasing and the microscopic orientation of the spins in the AFM layer. Early theories ${ }^{1,8,9}$ assumed that the AFM spins at the interface couple collinearly to the FM layer. Alternately, $\mathrm{Koon}^{10}$ has suggested that the AFM and FM spins can align perpendicular depending on the interfacial structure. Takano, et al. ${ }^{5}$ observed a correlation between the thermoremanent moment of polycrystalline $\mathrm{CoO} / \mathrm{MgO}$ multilavers and the exchange biasing field of $\mathrm{CoO} /$ permalloy bilayers. This result suggested that uncompensated interfacial spins are the principal origin of exchange biasing in that system. Unfortunately. it is difficult to determine the AFM spin structures involved in exchange biasing because few experimental techniques can probe AFM order in buried and interfacial layers.

The scope of the present experiment is to determine the orientation of the AFM moments relative to the $\mathrm{FM}$ spins in $(111) \mathrm{Co}(7.5 \mathrm{~nm}) / \mathrm{CoO}(\mathrm{X} \mathrm{nm})$ thin films $(\mathrm{X}=20,40$ and 100 nm) using polarized neutron diffraction methods. After field cooling our epitaxial samples, we find that the percentage of $\mathrm{CoO}$ spins perpendicular to the field exceeds the percentage of parallel spins. The difference between the parallel and perpendicular AFM moment projections in the $\mathrm{Co} / \mathrm{CoO}$ bilayers appears to be induced by proximity to the FM layer. This behavior may arise from perpendicular coupling between the $\mathrm{Co}$ and $\mathrm{CoO}$ spins, as predicted by Koon. ${ }^{10}$

For this study, the $\mathrm{CoO}$ films were reactively sputtered at $100{ }^{\circ} \mathrm{C}$ in 2 mTorr of $\mathrm{Ar}$ and a small partial pressure of oxygen (0.09 mTorr) onto 1"-diameter (0001) $\mathrm{Al}_{2} \mathrm{O}_{3}$ substrates. We examined five single-layer and bilayer samples with differing compositions, $\mathrm{SiO}_{2}(10 \mathrm{~nm}) / \mathrm{CoO}(\mathrm{X}) / \mathrm{Al}_{2} \mathrm{O}_{3}(\mathrm{X}=40$ and $100 \mathrm{~nm})$ and $\mathrm{SiO}_{2}(10 \mathrm{~nm}) / \mathrm{Co}(7.5 \mathrm{~nm}) / \mathrm{CoO}(\mathrm{X}) / \mathrm{Al}_{2} \mathrm{O}_{3}(\mathrm{X}=20,40$ and $100 \mathrm{~nm})$. In order to induce 
exchange-biasing and uniaxial anisotropy in the FM layer, the $\mathrm{Co} / \mathrm{CoO}$ bilayers were deposited in a 300 Oe field applied along the $[11 \overline{2} 0] \mathrm{Al}_{2} \mathrm{O}_{3}$ in-plane axis. High-angle $\mathrm{x}$-ray diffraction confirms that the $\mathrm{CoO}$ layers are epitaxial with (111) preferred orientation and are twinned in the growth plane. ${ }^{11}$ Fits to $\mathrm{x}$-ray reflectivity data for the $\mathrm{Co} / \mathrm{CoO}(40 \mathrm{~nm})$ bilayer indicate that the width of the $\mathrm{Co} / \mathrm{CoO}$ interface is approximately $1.0 \mathrm{~nm}$ due to roughness or interdiffusion.

Magnetization measurements performed in a SQUID magnetometer reveal that exchangebiasing is observed for a comparable epitaxial $\mathrm{Co} / \mathrm{CoO}(100 \mathrm{~nm})$ bilayer grown under similar conditions on smaller substrates. Figures 1 (a) and (b) show hysteresis loops at 10 and 200 $\mathrm{K}$ respectively after cooling in a $10 \mathrm{kOe}$ field applied along the same direction as the growth field. Both the coercive field and the exchange-biasing field, $H_{e b}$, change as a function of temperature, as shown in Fig. 1. These field values are expected to differ for bilayers with thinner $\mathrm{CoO}$ and may depend on the film structure as determined by the substrate characteristics.

For these and related biased layers, ${ }^{12}$ the temperature marking the onset of biasing is nearly equal to the Néel temperature, $T_{N}$, of the AFM component. Bulk $\mathrm{CoO}$ orders as a collinear antiferromagnet below $T_{N}=291 \mathrm{~K}$. The spins are ferromagnetically aligned in (111) sheets and are nearly perpendicular to a $\langle 111\rangle$ axis. The moment direction alternates in neighboring (111) planes. ${ }^{13}$ The $\mathrm{CoO}$ spins can thus align in any of four equivalent $\{111\}$ domains. For our thin films, the [111] "propagation-axis" of one of these domains is parallel to the growth directions. Due to twinning, however, there are six additional propagation-axis tilted $70.5^{\circ}$ from the [111] growth direction. Complementary neutron diffraction studies ${ }^{14}$ revealed that a large portion of the $\mathrm{CoO}$ spins reside in these six off-axis domains. We restricted our study to the characterization of those spins in the (111) growth-axis domain, which give rise to a $(1 / 21 / 21 / 2)$ magnetic reflection in our neutron data.

To characterize the AFM structure associated with the biased state in the $\mathrm{Co} / \mathrm{CoO}$ bilayers, we performed polarized neutron diffraction measurements on these samples using the SPINS triple-axis spectrometer at the NIST Research Reactor. An electromagnet provided 
fields ranging from 50 to 2400 Oe. Since the maximum field is limited, we examined our samples at $200 \mathrm{~K}$, rather than $10 \mathrm{~K}$, in order to insure saturation of the Co layer upon field cooling (Fig. 1). On the SPINS spectrometer, polarizing elements were positioned before and after the sample to select the spin direction of the incident and scattered neutrons. The polarization efficiencies were approximately $92 \%$. For all scans through the $(1 / 21 / 2$ 1/2) $\mathrm{CoO}$ reflection, we measured both the non-spin-flip (NSF) and spin-flip (SF) scattered intensities. The NSF intensity is proportional to the square of the average $\mathrm{CoO}$ moment projection parallel to the vertical applied field, while the SF intensity is proportional to the square of the average moment projection perpendicular to the field. ${ }^{15}$ These moment projections may belong to the same or to different (111) domains.

Figure 2 shows typical scans through the $(1 / 21 / 21 / 2)$ reflection for the $\mathrm{Co} / \mathrm{CoO}(100$ $\mathrm{nm}$ ) bilayer at $200 \mathrm{~K}$. After cooling the sample in zero field, the NSF and SF intensities are approximately equal [Fig. 2 (a)]. These results are consistent with random orientation of the $\mathrm{CoO}$ spins within the growth plane. Upon cooling in a -2200 Oe field and measuring in a 2200 Oe field, the SF intensity increases slightly relative to the NSF as shown in Fig. 2 (b). To determine if this difference is significant, we counted the NSF and SF intensities at the center of the $(1 / 21 / 21 / 2)$ reflection for extended times. Upon demagnetizing and zero field cooling, the ratio of the NSF to SF intensity is $1.001 \pm 0.011$, compared to a ratio of $0.941 \pm 0.017$ for the same field preparation as in Fig. 2 (b). However, a single-layer $\mathrm{CoO}(100 \mathrm{~nm})$ film also gives a NSF/SF ratio of $0.94 \pm 0.012$ upon field cooling in $2400 \mathrm{Oe}$ (Fig. 3). The AFM spins in the $\mathrm{Co} / \mathrm{CoO}(100 \mathrm{~nm})$ bilayer do not clearly show any effects from coupling to the FM Co layer. Since the data in Fig. 2 represent the $\mathrm{CoO}$ spin configuration averaged both along the growth axis and across the growth plane, the direction of the $\mathrm{CoO}$ moments near the $\mathrm{Co} / \mathrm{CoO}$ interface can not be directly distinguished from the direction of those moments in the remainder of the $100 \mathrm{~nm}$ layer. Any local deviations of the CoO spin direction at the $\mathrm{Co} / \mathrm{CoO}$ interface may be masked by the bulk behavior of the thick AFM layer.

To determine if the perpendicular AFM spins are close to the interface, we measured the 
$(1 / 21 / 21 / 2) \mathrm{NSF}$ and SF peak intensities for $\mathrm{Co} / \mathrm{CoO}$ samples with thinner $\mathrm{CoO}$ layers. Initially cooling in zero field, the $\mathrm{Co} / \mathrm{CoO}(40 \mathrm{~nm})$ bilayer shows a tendency for perpendicular spin alignment $(\mathrm{NSF} / \mathrm{SF}=0.933 \pm 0.01)$. Upon field cooling and measuring in $2400 \mathrm{Oe}$, the NSF/SF ratio of $0.873 \pm 0.01$ is significantly smaller than that for the $\mathrm{Co} / \mathrm{CoO}(100 \mathrm{~nm})$ sample, as shown in Fig. 3. This ratio varies only slightly up to $0.895 \pm 0.011$ when the field is lowered to 150 Oe. Thus this spin configuration appears to "freeze-in" upon field cooling. The average perpendicular projection of the $\mathrm{CoO}$ moment is at least $3 \%$ larger than the parallel projection. For the $\mathrm{Co} / \mathrm{CoO}(20 \mathrm{~nm})$ bilayer, a NSF/SF ratio of $0.928 \pm$ 0.017 was measured in 2300 Oe after field cooling. This value is comparable to that for the $\mathrm{Co} / \mathrm{CoO}(40 \mathrm{~nm})$ bilayer. As shown in Fig. 3, the $\mathrm{Co} / \mathrm{CoO}(20 \mathrm{~nm})$ and $\mathrm{Co} / \mathrm{CoO}(40 \mathrm{~nm})$ films have a larger fraction of perpendicular spins than the $\mathrm{Co} / \mathrm{CoO}(100 \mathrm{~nm})$ film. This result may indicate that the canted spins are confined primarily to a finite region near the $\mathrm{Co} / \mathrm{CoO}$ interface. For a more careful check of this hypothesis, the NSF/SF ratio should be accurately measured over the entire $Q$ range of the Bragg reflection (e.g., Fig. 2).

Overall, these data suggest that the $\mathrm{CoO}$ spins in the biased bilayers prefer a perpendicular orientation relative to the cooling field. However, this alignment could either be a consequence of the $\mathrm{Co} / \mathrm{CoO}$ interlayer coupling or a direct response to the cooling field. To distinguish between these two effects, we performed field-dependent diffraction measurements on a $\mathrm{CoO}(40 \mathrm{~nm})$ film. Upon field cooling and measuring the sample in a $2300 \mathrm{Oe}$ field, the ratio of the NSF to $\mathrm{SF}$ intensities for the $(1 / 21 / 21 / 2)$ reflection is $1.02 \pm 0.017$, which is significantly greater than the value measured for the corresponding $\mathrm{Co} / \mathrm{CoO}(40 \mathrm{~nm})$ bilayer (Fig. 3). The random orientation of the AFM spins is apparently unperturbed by cooling fields up to $2400 \mathrm{Oe}$. The preferred perpendicular orientation observed in $\mathrm{Co} / \mathrm{CoO}$ bilayers thus appears to result from interlayer coupling. The following measurement for the $\mathrm{Co} / \mathrm{CoO}(40 \mathrm{~nm})$ bilayer further supports this conclusion. We first cooled the sample to 200 $K$ in a 2400 Oe field applied in-plane parallel to the Co growth field direction. We lowered the field to zero and then applied an in-plane field of 500 Oe at an angle of $90^{\circ}$ relative to the field cooling direction while maintaining a temperature of $200 \mathrm{~K}$. The resultant NSF/SF 
ratio is $1.02 \pm 0.011$, which is larger than the $0.873 \pm 0.01$ ratio obtained in a $2400 \mathrm{Oe}$ after initially field cooling along the easy axis (Fig. 3). The "frozen" $\mathrm{CoO}$ spins thus try to align perpendicular to the reoriented Co layer moment. Since the 500 Oe field is too small to rotate the $\mathrm{CoO}$ spins, the moment reorientation is a direct response to the change in the Co magnetization direction.

In conclusion, we observe a preferential orientation of the $\mathrm{CoO}$ moments away from the Co magnetization direction in (111) $\mathrm{Co} / \mathrm{CoO}$ bilayers. Within the (111) growth-axis domain, the perpendicular projection of the $\mathrm{CoO}$ moment relative to the field direction is larger than the parallel projection for the three bilayers considered. This result is consistent with Koon's model, ${ }^{10}$ which predicts $90^{\circ}$ coupling between the FM and AFM layers. The preferred perpendicular orientation of the AFM develops when the $\mathrm{Co} / \mathrm{CoO}$ bilayer is field cooled through the AFM $T_{N}$ into the biased state. Thus the $\mathrm{CoO}$ spin reorientation films appears to be a direct consequence of exchange coupling between the $\mathrm{Co}$ and $\mathrm{CoO}$ layers. Unfortunately, we can not as yet determine if the perpendicular spins reside in the interfacial region, or if the spins in the off-axis $\{111\}$ domains play an important role. By controlling the AFM spin population in the growth-axis and off-axis domains, future diffraction studies of related $\mathrm{CoO}$ films may indeed provide a complete understanding of the physical origin of exchange biasing.

This work is based upon activities supported by the National Science Foundation under Agreement \# DMR-9423101. The work of G.P.F. was supported by USDOE-BES under contract \#W 31-109-ENG-38. We appreciate helpful discussions with R.W. Erwin. 


\section{REFERENCES}

${ }^{1}$ W.H. Meiklejohn and C.P. Bean, Phys. Rev. 102, 1413 (1956); 105, 904 (1957).

${ }^{2}$ M.J. Carey and A.E. Berkowitz, Appl. Phys. Lett. 60, 3060 (1992).

${ }^{3}$ R. Jungblut, R. Coehoorn, M.T. Johnson, J. ann de Stegge and A. Reinders, J. Appl. Phys. 75, 6659 (1994).

${ }^{4}$ J. Nogues, D. Lederman, T.J. Moran, I.K. Schuller and K.V. Rao, Appl. Phys. Lett. 68, 3186 (1996); J. Nogues, D. Lederman, T.J. Moran and I.K. Schuller, Phys. Rev. Lett. 76, $4624(1996)$.

${ }^{5}$ Kentaro Takano, R.H. Kodama, A.E. Berkowitz, W. Cao and G. Thomas, Phys. Rev. Lett. $79,1130(1997)$.

${ }^{6}$ Y. Ijiri, J.A. Borchers, R.W. Erwin, S-H Lee, P.J. van der Zaag and R.M. Wolf, Phys. Rev. Lett. (submitted); J. Appl. Phys. (this conference).

${ }^{7}$ B. Dieny, V.S. Speriosu, S.S.P. Parkin, B.A. Gurney, D.R. Wilhout and D. Mauri, Phys. Rev. B 43, 1297 (1991).

${ }^{8}$ D. Mauri, H.C. Siegmann, P.S. Bagus and E. Kay, J. Appl. Phys. 62, 3047 (1987).

${ }^{9}$ A.P. Malozemoff, Phys. Rev. B37, 7673 (1988); J. Appl. Phys. 63, 3874 (1988).

${ }^{10}$ N.C. Koon, Phys. Rev. Lett. 78, 4865 (1997).

${ }^{11}$ M.J. Carey, F.E. Spada, A.E. Berkowitz, W. Cao and G. Thomas, J. Mater. Res. 6, 2680 (1991).

${ }^{12}$ M.J. Carey, A.E. Berkowitz, J.A. Borchers and R.W. Erwin, Phys. Rev. B47, 9952 (1993).

${ }^{13}$ W.L. Roth, Phys. Rev. 110, 1333 (1958); D. Herrmann-Ronzaud, P. Burlet and J. RossatMignod, J. Phys. C 11, 2123 (1978).

${ }^{14}$ J.A. Borchers, M.J. Carey, R.W. Erwin, C.F. Majkrzak and A.E. Berkowitz, Phys. Rev. 
Lett. 70, 1878 (1993).

${ }^{15}$ R.M. Moon, T. Riste and W.C. Koehler, Phys. Rev. 181, 920 (1969). 


\section{FIGURES}

FIG. 1. Magnetization as a function of field for a $\mathrm{Co} / \mathrm{CoO}(10 \mathrm{~nm})$ bilayer at (a) $10 \mathrm{~K}$ and (b) $200 \mathrm{~K}$ after field cooling from room temperature in a $10 \mathrm{kOe}$ field applied along the in-plane easy axis.

FIG. 2. Polarized neutron diffraction scan through the $(1 / 21 / 21 / 2)$ reflection for a $\mathrm{Co} / \mathrm{CoO}(10$ $\mathrm{nm}$ ) bilayer at $200 \mathrm{~K}$. The wavevector $Q$ is defined as $Q=\frac{4 \pi}{\lambda} \sin \theta$ where the neutron wavelength $\lambda=0.417 \mathrm{~nm}$ and $\theta$ is the scattering angle. The shaded circles represent the non-spin-flip (NSF) intensity and the open circles mark the spin-flip (SF) intensity. The data in (a) were obtained after demagnetizing the sample and then cooling in zero field (ZFC). The data in (b) were obtained in a 2200 Oe field after field cooling (FC) in -2200 Oe from room temperature. These data have not been corrected for the efficiencies of the polarizing elements.

FIG. 3. Ratio of the non-spin-flip (NSF) to spin-flip (SF) peak intensities for the $(1 / 21 / 21 / 2)$ $\mathrm{CoO}$ magnetic reflection as a function of $\mathrm{CoO}$ layer thickness for several $\mathrm{Co} / \mathrm{CoO}$ and $\mathrm{CoO}$ samples, as marked. The samples were cooled to $200 \mathrm{~K}$ in a $2200-2400 \mathrm{Oe}$ field and then measured in the same field. These data have not been corrected for background contributions or for the efficiencies of the polarizing elements. Background corrections would increase the ratio for the thinnest samples with the weakest intensities. 
F: 1 , Borchers, JAP, G0.06

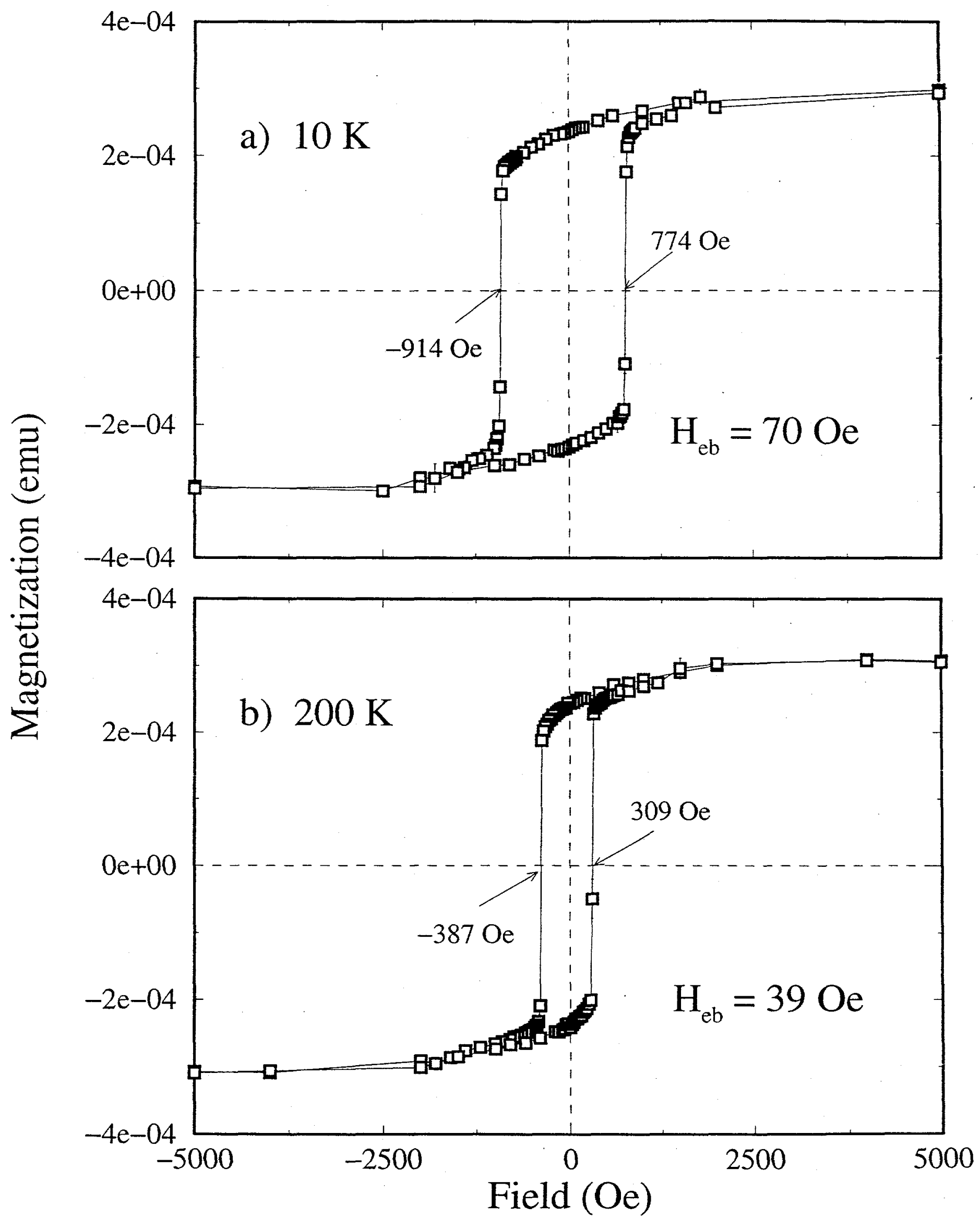


Figg, Borchers, JAP, GD-06

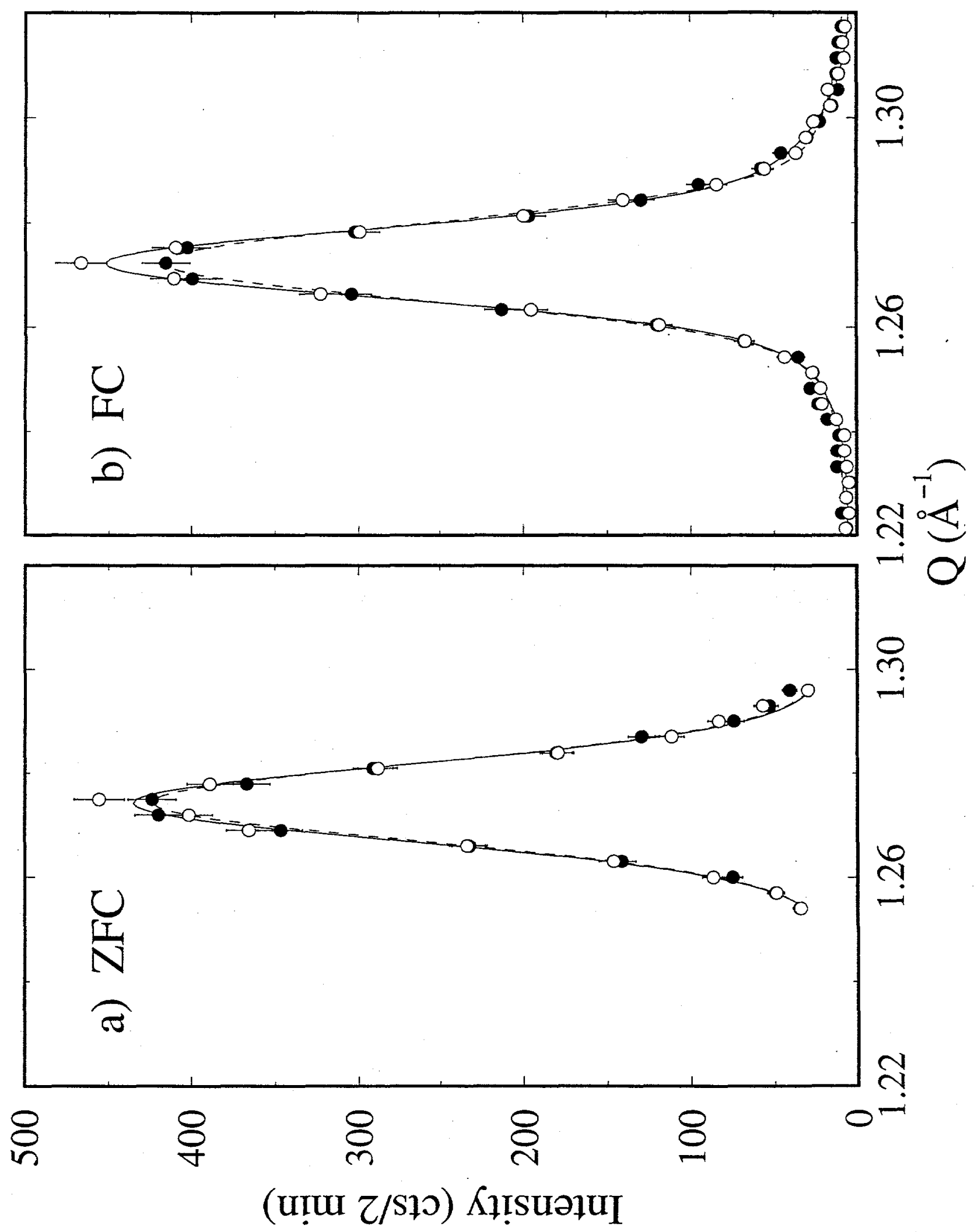


Fig3, Borchers, JAP, G0-06

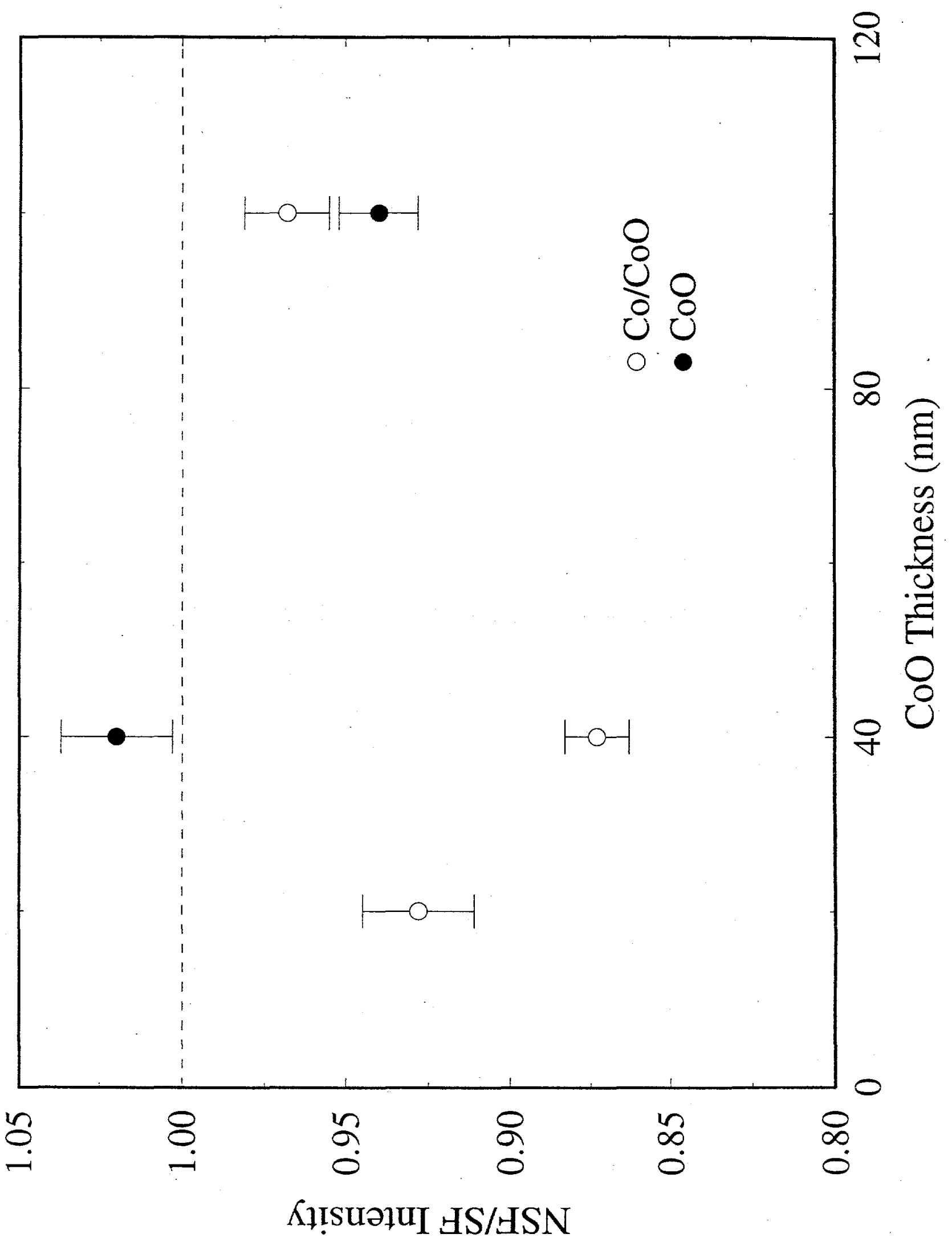

